\title{
Saliency Detection with VORONOI Diagram
}

\author{
Dao Nam Anh \\ Department of Information Technology \\ Electric Power University \\ 235 Hoang Quoc Viet road \\ Hanoi, Vietnam
}

\author{
Nguyen Huu Quynh \\ Department of Information Technology \\ Electric Power University \\ 235 Hoang Quoc Viet road \\ Hanoi, Vietnam
}

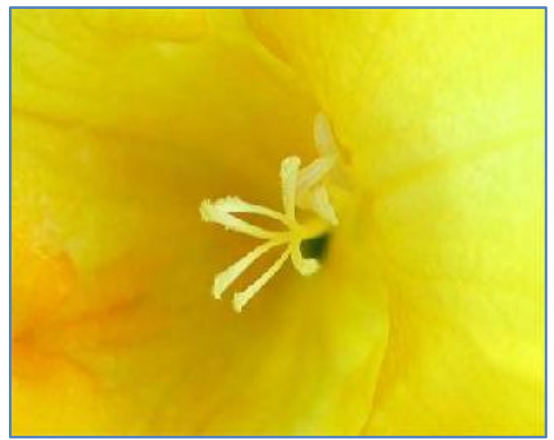

a. Input image

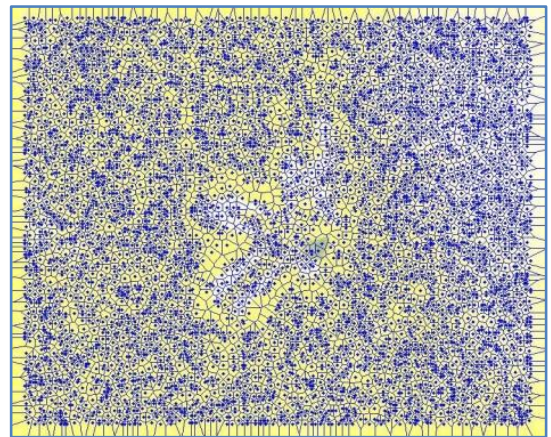

b. Voronoi diargram

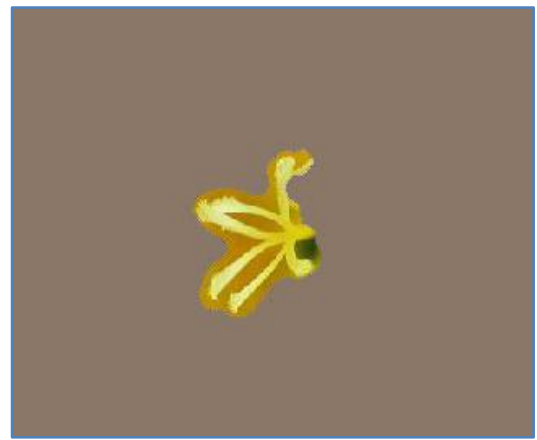

c. Salient region

Figure 1: Example of saliency detection with the Voronoi diagram

\begin{abstract}
Many applications are serviced by the Voronoi tessellation required to split image into Voronoi regions. An automatic method to learn and detect salient region for color image with support of the Voronoi diagram is presented. Salient regions are modeled as flexible circumstance corresponding to centers of mass. The centers are predicted by local contrast-based representation with local maxima. Results are demonstrated that are very competitive with other recent saliency map detection schemes and show robustness to capture visual attention objects. Our major contributions are the local maxima based method for allocation of Voronoi centroids and the Gaussian-based filter for estimating attention degrees. To show the effectiveness of the approach, saliency maps are detected for images of MSRA saliency object database by some state-of-the-art methods. The strengths and the weaknesses of the approach are considered, with a special focus on the context based salient regions - a challenging task which can be found in wide range of applications addressed in computer vision.
\end{abstract}

\section{General Terms}

Pattern Recognition, Algorithms.

\section{Keywords}

Voronoi tessellation, salient region.

\section{INTRODUCTION}

Computational geometry has attracted research interest in the past decade not least because of important application in, but not limited to, astronomy, biology, cartography, chemistry, forestry, linguistics, marketing, meteorology, physics, physiology and statistics. Voronoi diagrams [1][2] being a geometric construct of a discrete set of points and line segments have interesting and surprising properties in computational geometry and geometric modeling [3] and particularly in point pattern analysis [4].

The spectrum of the Voronoi diagram research for the image processing covers many areas including shape representation, fundamental segmentation, texture analysis, vector fields and special $k$-clustering. Features of Voronoi edges, vertices and circles generated by $k$-points can be used for optimum $k$ clustering [5] that provides solutions for color quantization and image compression [6]. A metric based on Voronoi regions of each point is used to define distance from a pixel to Voronoi points for image segmentation [7]. A particular Voronoi tessellation is addressed in [8], where the generators of the Voronoi regions in the tessellation are also the centers of mass. Thus, spatially distributed vector fields are clustered by a the Voronoi regions-based similarity measure function in both the spatial and vector spaces.

Voronoi tessellation is the base for a texture segmentation algorithm [9], where feature vector is calculated for each Voronoi polygon and then used to identify the interior and the border regions of textures. Voronoi skeletons in form of diagram of a polygon are constructed in [10] to derive accurate and robust skeletons for planar shapes. The skeletons possess the properties of connectivity as well as Euclidean metrics in the method.

Besides, visual saliency [11] is of high interest in many computer vision applications, ranging from image segmentation [12], content-aware compression [13], object detection [14] in web images [15] and focus of attention in human robot interaction [16].

One of our motivations for this research is to construct saliency map (example in Fig.1c) with advantages of Voronoi diagram (Fig.1b) in presenting image features such as contrast by computational geometry. This paper introduces a special method of creating Voronoi tessellation for saliency detection purpose. The Voronoi points are defined from analysis of local contrast. A local maxima filter is described for building Voronoi region.

To the best of our knowledge, an algorithm for detection of visual attention region is demonstrated with an standard benchmark designed for salient objects. As shown in an implementation, comparing with a range of different methods, this algorithm achieves respectable performance on the 
standard benchmark, and exhibits graceful ability to select detect salient regions.

\section{OUTLINE OF PAPER}

Section 3 reviews the Voronoi diagram and saliency detection research that relates to our method. Section 4 describes the proposed approach. Experiment results are reported in Section 5 , followed by discussion, directions for future work and conclusion in Section 6, 7, 8 .

\section{PRIOR WORK}

This section first reviews methods aiming for image segmentation using Voronoi tessellation. Then, methods that apply the Voronoi diagram to detect salient region/object are reported.

\subsection{VORONOI SEGMENTATION}

Segmentation of human faces is addressed by Voronoi Diagram approach [17] that applies a distance transformation to segment face features. The approach employs a greedy search algorithm looking for a particular face candidate by focusing its action in elliptical-like regions. A segmentation method by region growing is presented in [18], where seed points are derived from the color edge image as the peaks in the associated Voronoi diagram. Regions are grown using gates on pixel color relative to region central color and region edge pixel color.

Voronoi graph is used for autonomous place detection that is sensor and domain independent [19]. The skeleton of the free space in the local surround is found by a real-time calculated Voronoi graph. An algorithm based on the edge-weighted centroid Voronoi tessellation which uses propagation of the inter-slice consistency constraint is presented in [20]. It can segment a 3D superalloy image, slice by slice, to obtain the underlying grain microstructures.

An interactive image segmentation method based on structural pattern recognition is introduced in [21]. A model graph is constructed from an over-segmentation of an image and from traces given by user. The over-segmentation is controlled by markers provided by centroidal Voronoi diagrams.

\subsection{VORONOI SALIENCY}

Generalized Voronoi tessellation is outlined in low-level segmentation for vector valued images [22] where segmentation procedure uses an accordant pseudo-metric from the image data and the choice of a set of sources. The method predesigns saliency image which is related to the contrast ultra-metric and then estimation of the edges is based on the metric.

An iterative segmentation voting method, which uses oriented kernels for deriving visual attention as it relates to symmetry is outlined in [23]. Voronoi tessellation, voting, and level set methods are joined to outline blob-like structures in the method, implemented for biological data.

Perceptual grouping and domain meshing are addressed in [24]. The article shows a solution for the problems by Voronoi diagrams and their dual Delaunay complexes, defined with geodesic distances over 2D Riemannian manifolds.

The Voronoi diagram, traditionally used for point patterns, is applied to define region neighbors in [25] for an object description. The generalized Voronoi diagram partitions an image into polygons, each covering a region, representing its area of influence.
A segmentation method for multi-regions is carried out in two phases using the Voronoi supports at vertices and the implicit integration is demonstrated in [26]. The volume belonging to each patch segment can be achieved with the support of Voronoi diagram computed in the voxel space.

\section{VORONOI SALIENCY DETECTOR}

Denote color image $u(x)$ on space $\Omega: u(x): \Omega \rightarrow \mathfrak{R}^{3}$

Saliency map $s(x)$ encodes local visual attention:

$s(u): \Omega \rightarrow[0,1] \in \mathfrak{R}^{1}$

Voronoi diagram $V$ divides space $\Omega$ into a number of cells $C$ for a set of seed generators $G . V=\{G, C\}$

The generators $G$ are predefined by function $g$ :

$$
\begin{aligned}
& \left.G=\left\{z_{i}\right\}_{i=1}^{k}, z_{i} \in G \subset \Omega\right\} \\
& g(z)=\left\{\begin{array}{c}
g(z)=\text { true }, z \in G \\
g(z)=\text { false }, z \in \Omega \backslash G
\end{array}\right.
\end{aligned}
$$

There is one Voronoi cell for each seed generator:

$$
\begin{aligned}
& \operatorname{cell}\left(z_{i}\right): G \rightarrow C, \operatorname{cell}\left(z_{i}\right)=C_{i} \\
& \text { generator }\left(C_{i}\right): C \rightarrow G, \text { generator }\left(C_{i}\right)=z_{i}
\end{aligned}
$$

A Voronoi cell or tessellation consists of all pixels closer to that seed than any other:

$$
\begin{aligned}
& C=\left\{C_{i}\right\}_{i=1}^{k}, C_{i} \subset \Omega, \forall i \in\{1, . . k\} \\
& C_{i} \cap C_{j}=\varnothing, \forall i \neq j, \bigcup_{i=1}^{k} C_{i}=\Omega \\
& C_{i}=\left\{z \in C_{i},\left|z-z_{i}\right|<\left|z-z_{j}\right|, \forall z_{j} \subset G, j=1, . . k, j \neq i\right\}
\end{aligned}
$$

Creating Voronoi cells from Voronoi generators is noted by

$\operatorname{Voronoi}(G): G \rightarrow C$

\subsection{LOCAL MAXIMA FILTER}

To detect Voronoi seed generators a Gaussian-based local maxima filter is described as follows:

$$
f(\mathrm{x})=d\left(\operatorname{agrmax}_{y \in N(x) \cap G} \frac{1}{\sigma \sqrt{2 \pi}} \exp \left(-\frac{\|y-x\|^{2}}{2 \sigma^{2}}\right) d(\mathrm{y})\right)
$$

The filter provides searching for a neighbor pixel $y \in N(x)$ that gets local relative maximum: $y$ near $x$ and the values $d(z)$ of $d$ for all neighbor of $y$ are all less than $d(y)$. Then assign value $d$ at $y$ to $d$ at $x$.

\subsection{LOCAL VARIATION}

Local variation of a pixel $\mathrm{x}$ with its neighbors $y \in N(x)$ is defined as follows:

$$
v(x)=\frac{1}{W} \sum_{y \in N(x)} \frac{1}{\sigma \sqrt{2 \pi}} \exp \left(-\frac{\|y-x\|^{2}}{2 \sigma^{2}}\right)\|u(x)-u(y)\|^{2}
$$

Local variation mean of a region is calculated from (11):

$$
m(x)=\frac{1}{\operatorname{numel}(N(x))} \sum_{y \in N(x)} v(y)
$$

Thus, divergence at a pixel $x$ is described by difference of the variation $v(x)$ over the mean variation $m(x)$ :

$d(x)=\|v(x)-m(x)\|^{2}$

Set of local maxima $M$ on $d(x)$ can be found from (13):

$\left.M=\left\{x_{j}\right\}, x_{j} \in \Omega\right\}$ 
where $d(x)$ achieves local maxima at $x_{i}$ over all its neighbors $y \in N(x)$

$d\left(x_{i}\right)=\max _{y \in N\left(x_{i}\right)} d(y), x_{i} \in M$

The Voronoi generators $G(4)$ is found by the set of local maxima $M: G:=M$

\subsection{SALIENT CELLS}

To find salient regions the Voronoi cells with their divergence $d(x)$ are implemented. Salient value for the pixel $x$ that is a generator $\left(x=z_{i}\right)$ is the same its divergence:

$s(x):=d(x)$

For other pixel $x$, find corresponding generator $z_{i}$ by the local maxima filter $f(x)(10)$ :

$z_{i}=f(x)$

then assign salient value $d\left(z_{i}\right)$ for $s(x)$ :

$s(x)=d(f(x))=d\left(z_{i}\right)$

Salient map $s(x)$ is continuous, with the Otsu threshold:

$o(s): \operatorname{histogram}(o) \rightarrow \tau$

salient region can be detected:

$$
\begin{aligned}
& \Omega^{s}=\{x: s(x) \geq \tau\} \\
& \Omega^{b}=\Omega \backslash \Omega^{s}
\end{aligned}
$$

\subsection{VORONOI SALIENCY ALGORITHM}

We now elaborate above local maxima filter and Voronoi diagram into the Voronoi Saliency Algorithm (VSA).

Start: given an input image $u(x), \sigma$

1.Local variation calculation: define local variation $v(x)$ and divergence $d(x)$ by (11)(13).

2.Local Maxima detection: use (14)(15).

3.Voronoi diagram creation: apply (16)(18).

4.Saliency map: get $s(x)$ by saliency filter (17)(19).

5.Thresholding and export output by (20)(21).

Figure 2 illustrates the VSA algorithm working with an image (Fig.2a) from MSRA Salient Object Database [27]. Step 1 produces local variation $v(x)$ showed in Fig.2a and the divergence $d(x)$ - Fig.1c. Step 3 creates Voronoi generators G (Fig.1d) and cells C (Fig.1e, Fig.1f). Step 4 gives saliency map $s(x)$ presented in Fig.1g, followed by step 5, that exports final extracted salient region (Fig.1f).

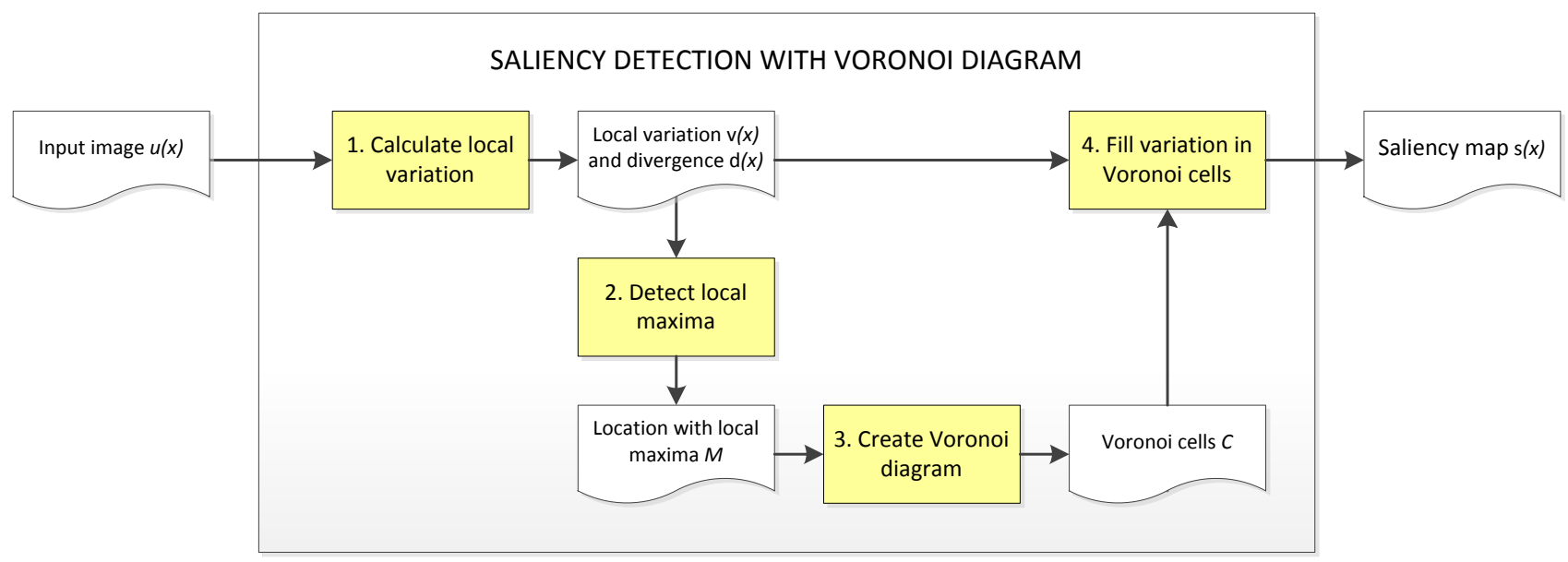

Figure 2: Algorithm of Saliency Detection with Voronoi Diagram

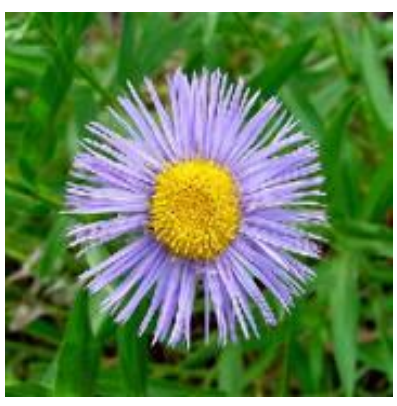

a) Input $u(x): 28 \_28988 . j p g$ [27]

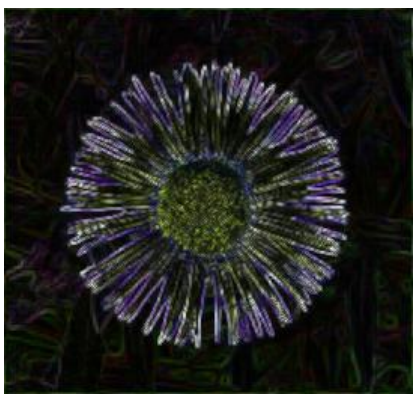

b) Local variation $v(x)$ by (11)

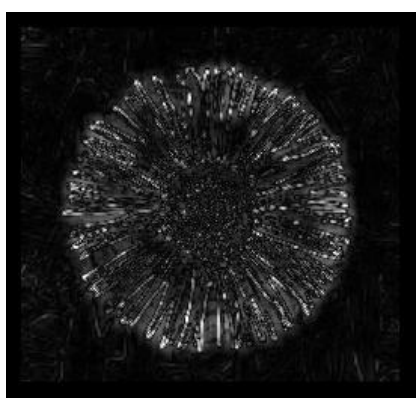

c) Divergence $d(x)$ by (13)

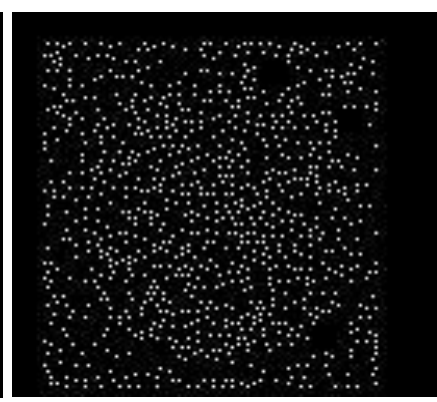

d) Local maxima $M$, generator $G$ 


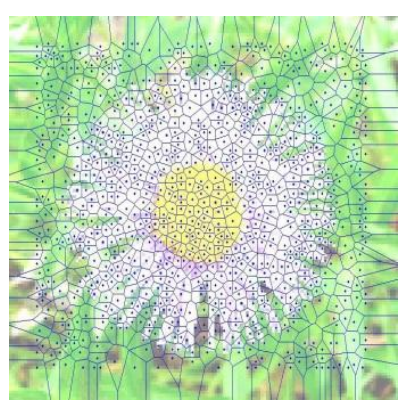

e) Voronoi cells $C$

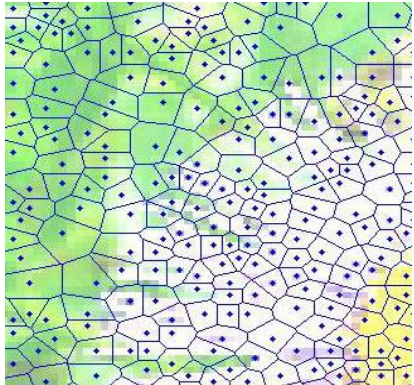

f) Voronoi cells $C$, zoom in

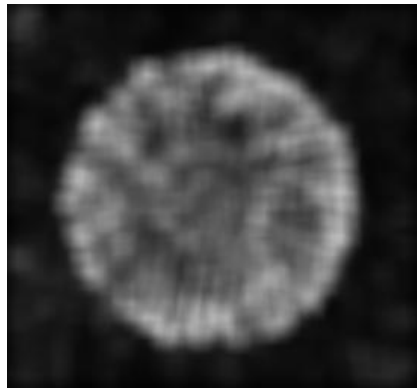

g) Saliency map $s(x)$ by (19)

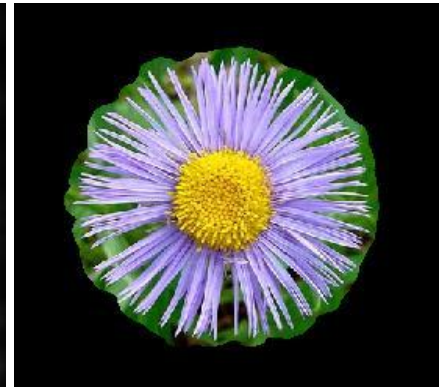

f) Extracted salient region $\Omega$ (21)

Figure 3: Example of saliency detection with the Voronoi diagram

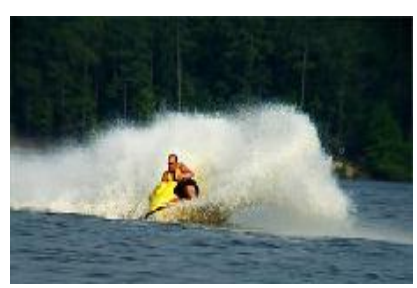

a) 27_27017.jpg

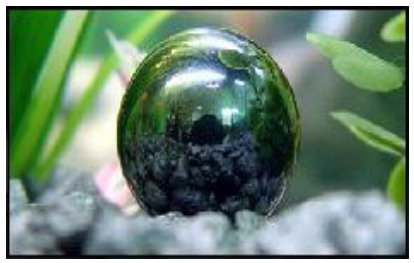

e) 28_28907.jpg

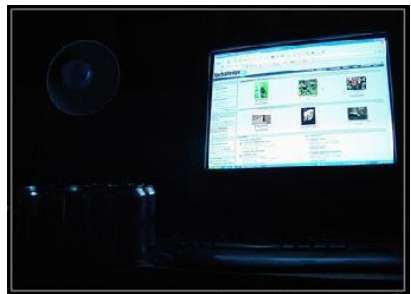

i) $28 \_28003 . . j p g$

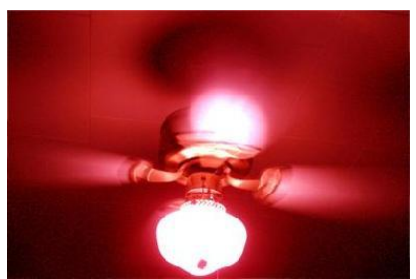

m) 27_27186.jpg

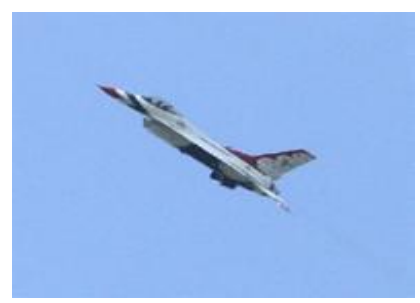

q) 27_27330.jpg

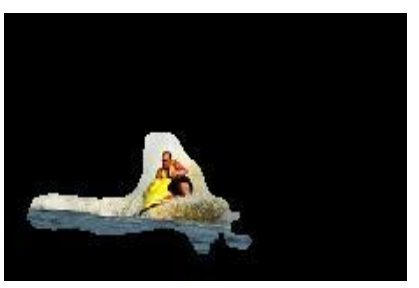

b) Extracted object

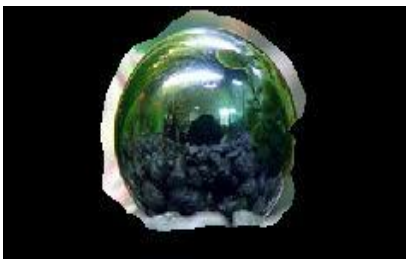

f) Extracted object

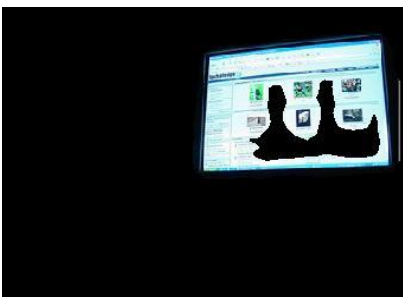

j) Extracted object

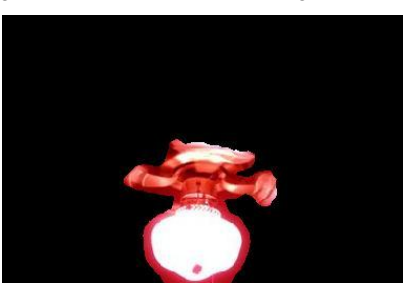

n) Extracted object

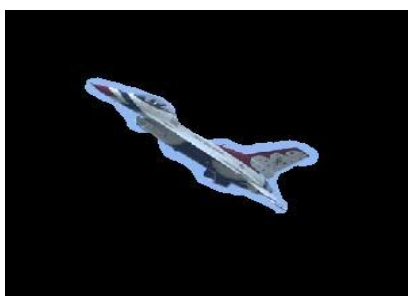

r) Extracted object

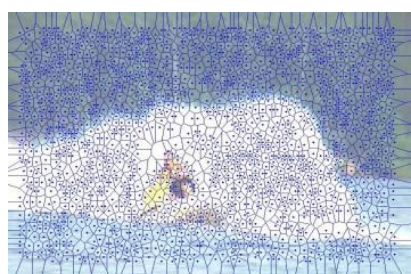

c) Voronoi diagram

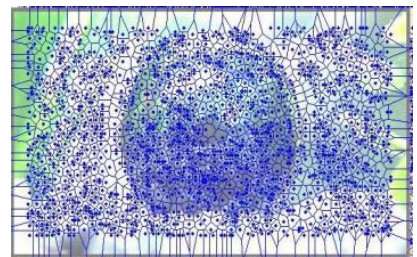

g) Voronoi diagram

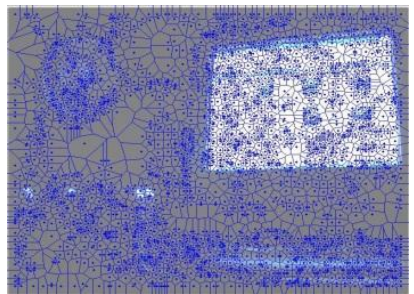

k) Voronoi diagram

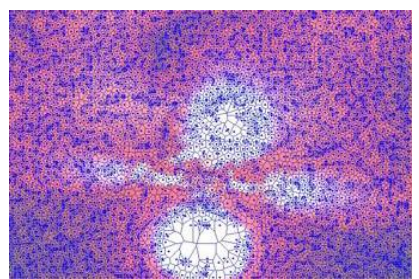

o) Voronoi diagram

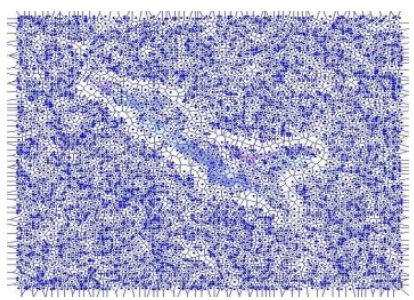

s) Voronoi diagram

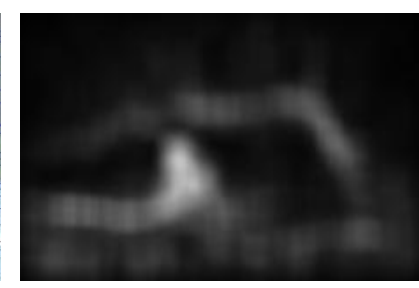

d) Saliency map

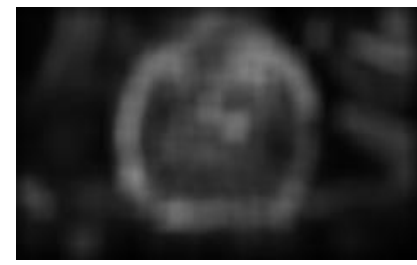

h) Saliency map

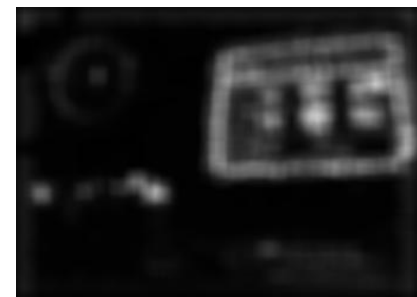

l) Saliency map

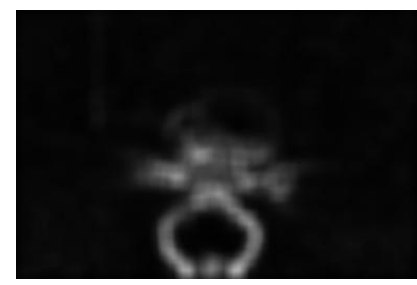

p) Saliency map

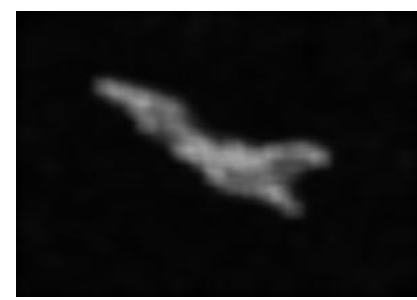

t) Saliency map 


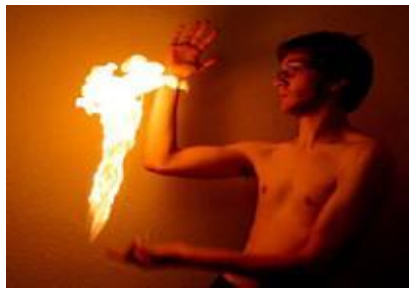

u) 28_28209.jpg

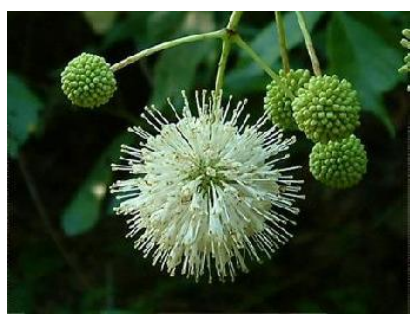

y) $28 \_28717 . j p g$

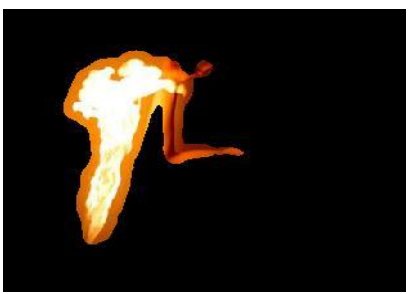

v) Extracted object

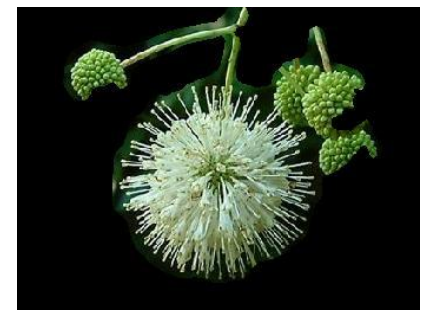

z) Extracted object

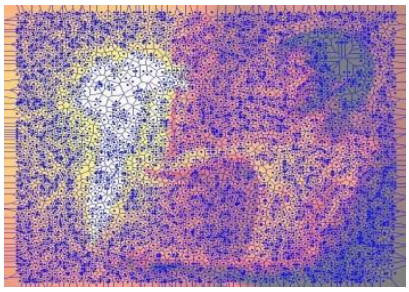

w) Voronoi diagram

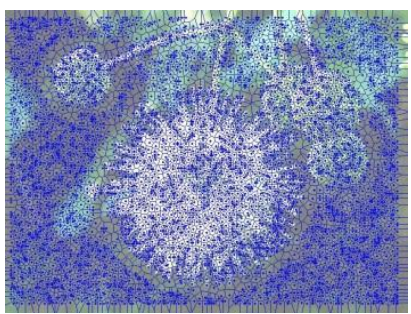

aa) Voronoi diagram

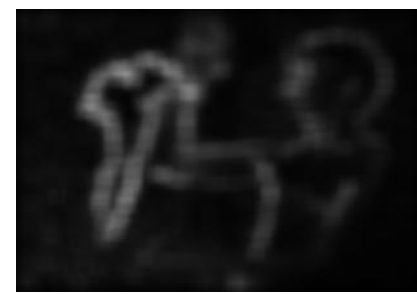

x) Saliency map

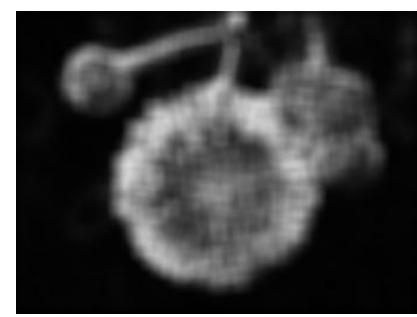

bb) Saliency map

Figure 4: Example of saliency detection with the Voronoi diagram for color images from [27]

\section{EXPERIMENTS}

Figure 3 shows experimental results of the saliency detection method with Voronoi diagram. Input images given by [27] are displayed in the first column. They belong to different image categories like nature, day and night. The Voronoi diagram which is calculated in VSA's the third step with generator and cells are presented in the third column. Saliency map produced by the fourth step for each test image is outlined in the fourth column.

Finally, salient region extracted by VSA's the fourth step is demonstrated in the second column next to its input. Note that the Voronoi generators $z_{i}$ are local maxima of divergence $d(x)$ and the saliency value $s(x)$ for each Voronoi cell $C_{i}$ is the divergence value at the generator $z_{i}$ of the cell.

Results of initial experiments on the MSRA Salient Object Database [27] are associated with the some state-of-the-art methods [28][29][30].

Rarity-based saliency detection [28] is sequential bottom-up features extraction. The features of the model are both lowlevel (luminance and chrominance) and medium-level (orientations).

Saliency Detection by Self-Resemblance [29] is a bottom-up saliency detection algorithm. This employs local steering kernels and applies a nonparametric kernel density estimation based on matrix cosine similarity.

Saliency For Image Manipulation [30] approach is based on four principles: pixel distinctness, pixel reciprocity, object association and multi-layer saliency. Basic saliency map is constructed from a distinctness map, based on the first and second principles, and a prominent object probability map, based on the third principle.

The VSA and these three methods are tested on a set of images of the salient object benchmark [27]. The database provides three user inputs for each test image. The inputs are in a form of rectangular covering region of visual attention. This format is not right for all the cases, particularly if a salient object is not fit well in a rectangular. Though, test with the database could give a relative performance comparison.

Mean squared error (MSE) and the sum of absolute differences (SAD) are selected metrics for this test. MSE measures the average of the squares of errors which is the difference between the estimator and the object for measurement. SAD weights the similarity between image blocks by taking the absolute difference between each pixel in the original block and the corresponding pixel in the block being used for comparison.

Examples of the results are displayed in Fig.7. Note that saliency map have a continuous range $[0,1]$.

Depending on rounding and thresholding methods, extraction of salient regions could be variable for the same input images. Though, the statistics from the experiment in Fig.6 demonstrate that the algorithm for saliency detection with Voronoi achieves competitive performance on this standard dataset.

\section{DISCUSSION}

The Voronoi diagram is suitable method to present salient degrees. In our algorithm the degree is based on contrast estimation expressed by variation and divergence for a local frame around each pixel. The size of frame and the Gaussian deviation are the main parameters of the algorithm. They can be chosen by statistics on performance for selected combinations of the parameters.

\section{FUTURE WORK}

The principle of Voronoi diagram is simple and effective. This article shows that the problem of saliency detection can be solved by the Voronoi diagram. Though our method is just one of several possible solutions. Further research on visual attention and Voronoi cells could open new results.

\section{CONCLUSION}

A method for detection saliency by Voronoi diagram is proposed by this paper. The method estimates divergence and local maxima on the divergence to build up the Voronoi generators. Tessellations are generated from the generators by a local maxima filter. The tessellations are the base for salient map.

The method is effective and simple to manage. It is tested at a standard saliency object database with a competitive performance on the MSE and SAD metrics.

\section{ACKNOWLEDGMENTS}

The authors would like to thank MSRA [27] for the database of salient objects. 
Special thanks authors of Rarity-based saliency detection [28], Saliency Detection by Self-Resemblance [29] and Saliency For Image Manipulation [30] for providing source codes that are used in our experimental implementation.

\begin{tabular}{|l|r|r|}
\hline Method & MSE & SAD \\
\hline $\begin{array}{l}\text { A. Rarity-based saliency detection } \\
\text { [28] }\end{array}$ & 0.15 & 0.24 \\
\hline $\begin{array}{l}\text { B. Saliency Detection by Self- } \\
\text { Resemblance [29] }\end{array}$ & 0.17 & 0.25 \\
\hline $\begin{array}{l}\text { C. Saliency For Image Manipulation } \\
\text { [30] }\end{array}$ & 0.15 & 0.23 \\
\hline $\begin{array}{l}\text { D. Saliency detection with Voronoi } \\
\text { diagram (VSA) }\end{array}$ & 0.14 & 0.23 \\
\hline
\end{tabular}

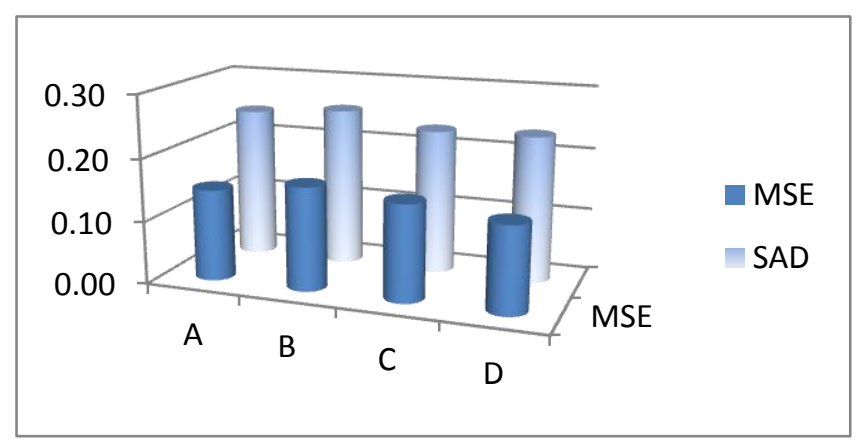

Figure 5: Statistics on experiment for saliency detection by methods [28][29][30] and VSA
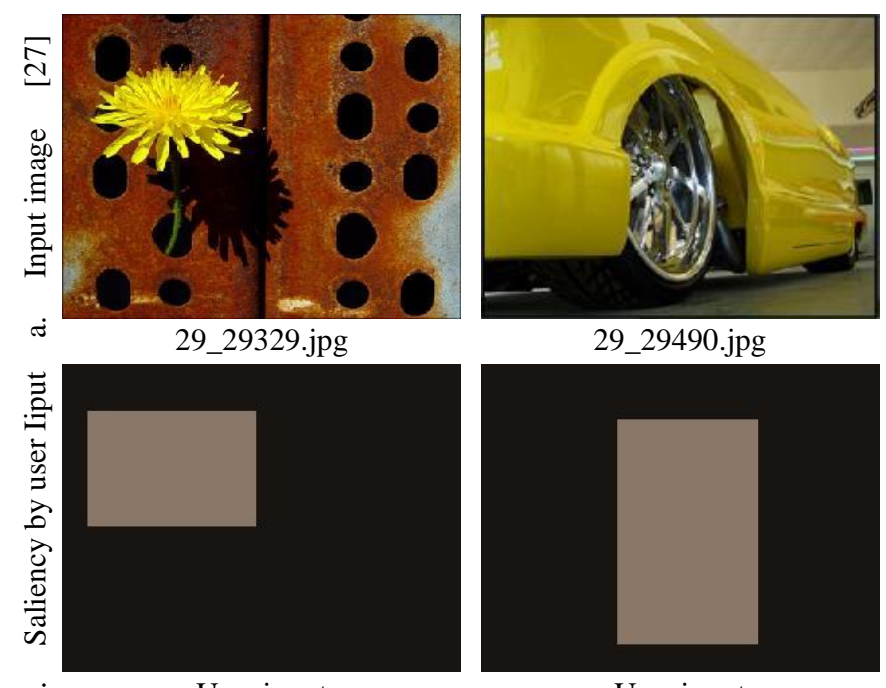

29_29490.jpg

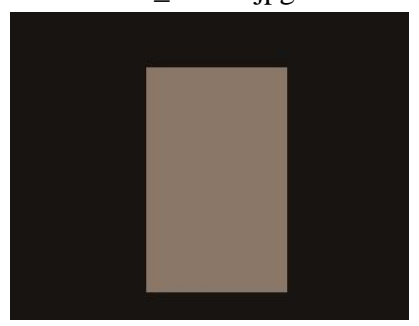

User input

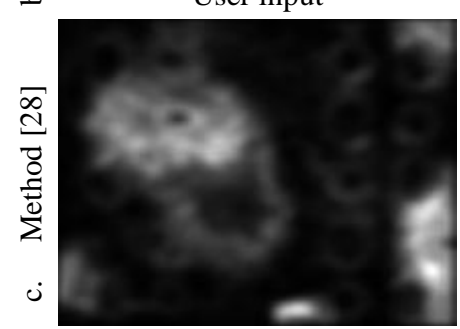

$\mathrm{MSE}=0.10, \mathrm{SAD}=0.20$

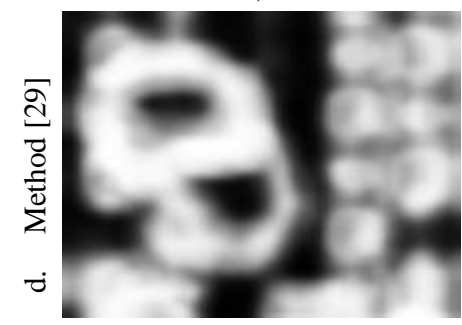

$\mathrm{MSE}=0.32, \mathrm{SAD}=0.47$

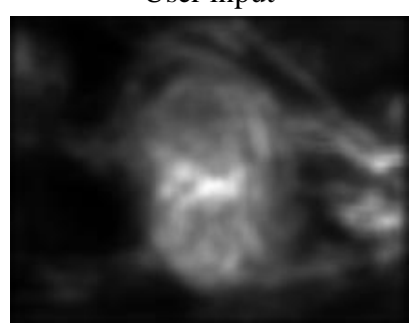

$\mathrm{MSE}=0.09, \mathrm{SAD}=0.21$

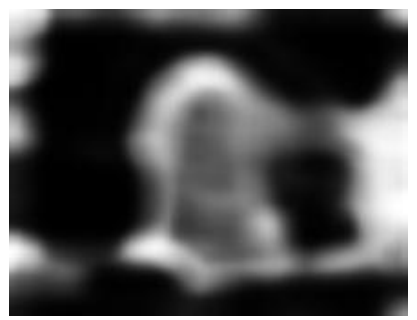

$\mathrm{MSE}=0.16, \mathrm{SAD}=0.28$

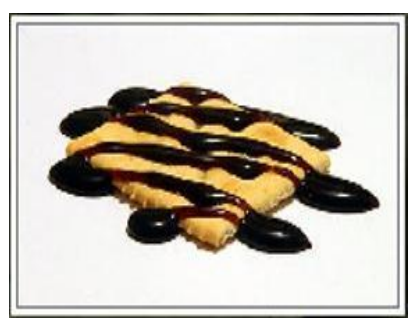

29_29729.jpg

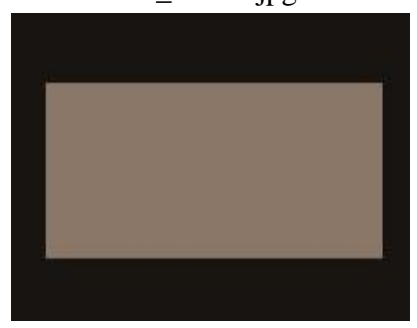

User input

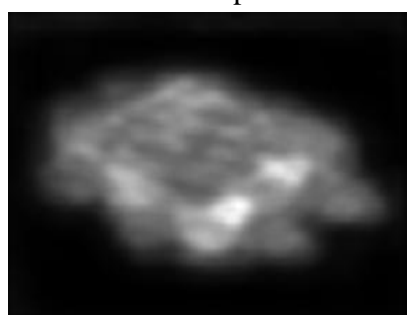

$\mathrm{MSE}=0.21, \mathrm{SAD}=0.32$

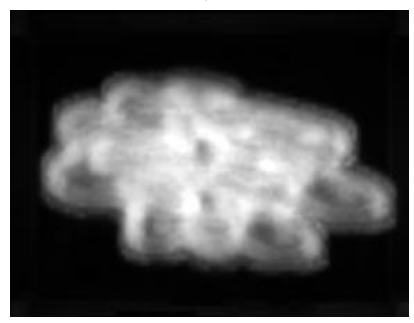

$\mathrm{MSE}=0.13, \mathrm{SAD}=0.20$

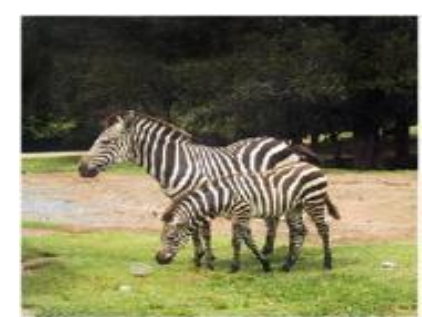

29_29788.jpg

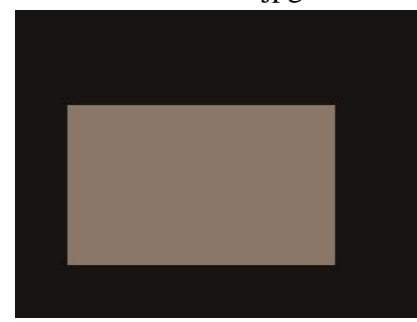

User input

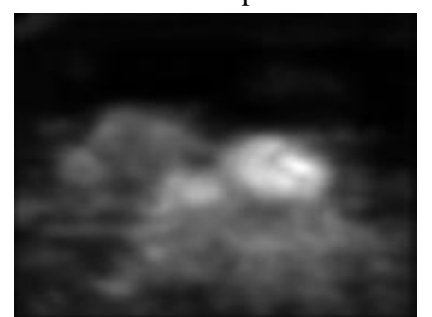

$\mathrm{MSE}=0.17, \mathrm{SAD}=0.29$

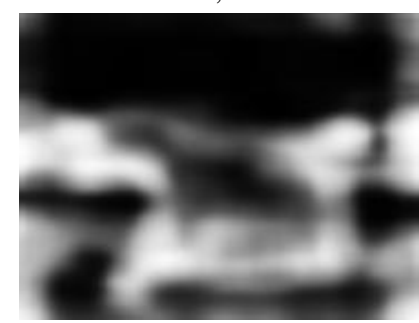

$\mathrm{MSE}=0.23, \mathrm{SAD}=0.36$ 


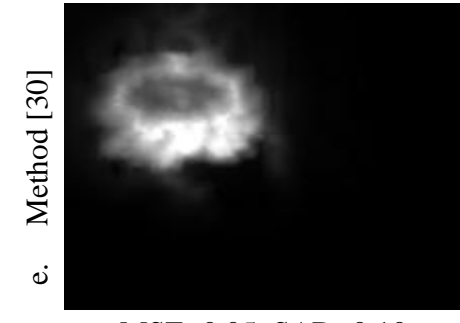

$\mathrm{MSE}=0.05, \mathrm{SAD}=0.10$

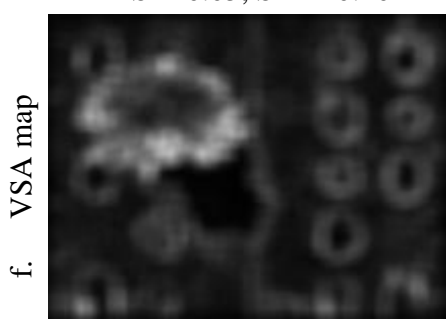

$\mathrm{MSE}=0.09, \mathrm{SAD}=0.21$

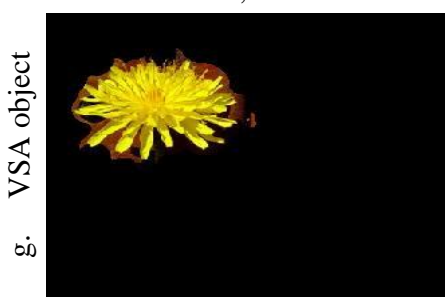

Figure 6: Example of saliency detection by methods [28][29][30] and VSA

\section{REFERENCES}

[1] G. Voronoi. Nouvelles applications des paramètres continus à la théorie des formes quadratiques. Journal für die Reine und Angewandte Mathematik, 133:97178,1907.

[2] Chew, L. P. 1986. Building Voronoi diagrams for convex polygons in linear expected time. Technical Report PCSTR90-147, Dept. Math. Comput. Sci., Dartmouth College, Hanover.

[3] Franz Aurenhammer, Voronoi diagrams - a survey of a fundamental geometric data structure, ACM computing surveys, vol.23, no.3, 1991, pages 345-405.

[4] Atsuyuki Okabe, Barry Boots, Kokichi Sugihara \& Sung Nok Chiu (2000). Spatial tessellations - concepts and applications of Voronoi diagrams. 2nd edition. John Wiley, 2000, 671 pages.

[5] S. Hasegawa, H. Imai, M. Inaba, N. Katoh, and J. Nakano, Efficient algorithms for variance-based kclustering, in Proceedings of the First Pacific Conference on Computer Graphics and Applications, World Scientific, River Edge, NJ, 1993, pp. 75-89.

[6] M. Inaba, H. Imai, and N. Katoh, Experimental results of randomized clustering algorithms, in Proceedings of the 12th ACM Symposium on Computational Geometry: Communication Section, 1996.

[7] Thouis R. Jones, A Carpenter, and P Golland. 2005. Voronoi-Based segmentation of cells on image manifolds. In Proc of the First inter. Conf. on Com. Vision for Biomed Image App. (CVBIA'05), pp 535-543.

[8] Q. Du and X. Wang, Centroidal Voronoi tessellation based algorithms for vector fields visualization and segmentation, Proc. IEEE Visualization, pp. 43-50, 2004.

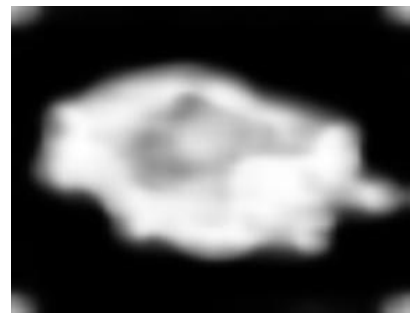

$\mathrm{MSE}=0.15, \mathrm{SAD}=0.24$

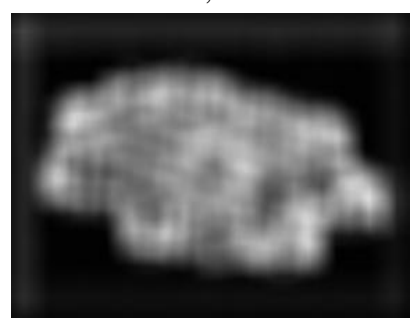

$\mathrm{MSE}=0.17, \mathrm{SAD}=0.31$

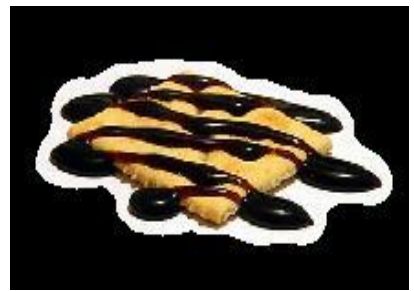

$\mathrm{MSE}=0.12, \mathrm{SAD}=0.22$

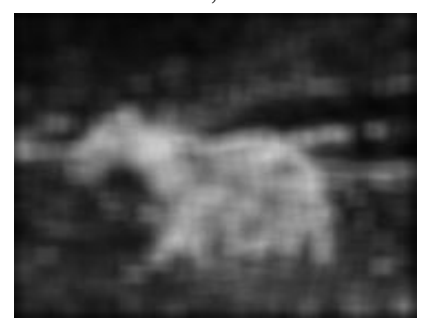

$\mathrm{MSE}=0.15, \mathrm{SAD}=0.31$

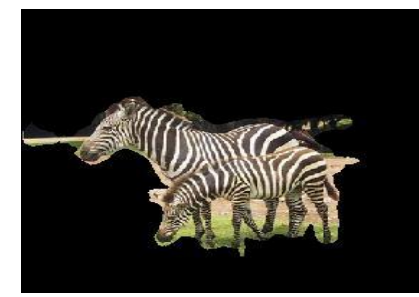

[9] ]M. Tüceryan and Anil K. Jain. 1990. Texture segmentation using Voronoi polygons. IEEE Trans. Pattern Anal. Mach. Intell. 12, 2 (1990), 211-216.

[10] Niranjan Mayya and V. T. Rajan. 1996. Voronoi diagrams of polygons: a framework for shape representation. J. Math. Im. Vis. 6, 4 (1996), 355-378.

[11] L. Itti, C. Koch, and E. Niebur. A model of saliencybased visual attention for rapid scene analysis. IEEE Trans. On PAMI, 20(11), 1998.

[12] J. Han, K. Ngan, M. Li, and H. Zhang. Unsupervised extraction of visual attention objects in color images. IEEE Transactions on Circuits and Systems for Video Technology, 16(1):141.145, 2006.

[13] Zund, F. , Pritch, Y. , Sorkine-Hornung, A. , Mangold, S., Content-aware compression using saliency-driven image retargeting, Image Processing (ICIP), 2013.

[14] A. Shokoufandeha, I. Marsicb, Sven J. Dickinsona, View-based object recognition using saliency maps, Image and Vision Computing 17 (1999) 445-460.

[15] P. Wang, J. Wang, G. Zeng, J. Feng, Salient object detection for searched web images via global saliency, (CVPR), 2012.

[16] G. Schillaci, S. Bodiroža, V. Hafner, Evaluating the effect of saliency detection and attention manipulation in human-robot interaction, Int. J. of Social Robotics, 2013, Vol. 5, Is. 1, pp 139-152.

[17] A. Cheddad, D. Mohamad and A. Abd Manaf, Exploiting Voronoi diagram properties in face segmentation and features extraction, Pattern Recognition, 41 (12) (2008) 3842-3859, Elsevier Science.

[18] Sinclair, D.: Voronoi seeded colour image segmentation. Technical Report 3, AT\&T Laboratories Cambridge (1999). 
[19] P. Beeson, N. K. Jong, and B. Kuipers, Towards autonomous topological place detection using the extended Voronoi graph, IEEE Inter. Conference on Robotics and Automaton (ICRA), 2005.

[20] Y. Zhou, L. Ju, Y. Cao, Jarrell W. Waggoner, Y. Lin, J. Simmons, S. Wang. Edge-weighted centroid Voronoi tessellation with propagation of consistency constraint for $3 \mathrm{~d}$ grain segmentation in microscopic superalloy images. CVPR Workshops 2014: 258-265.

[21] Narendra Ahuja, Sinisa Todorovic, Connected segmentation tree -a joint representation of region layout and hierarchy, Proc. IEEE Conf. Computer Vision and Pattern Recognition (CVPR), 2008.

[22] Arbelaez, P. A., \& Cohen, L.D. (2003). Generalized Voronoi tessellations for vector-valued image segmentation. Proc. $2^{\text {nd }}$ IEEE Workshop on Variational, Geometric and Level Set Methods in Computer Vision (VLSM'03) (pp.49-56).

[23] H. Chang, Q.Yang, B. Parvin, Segmentation of heterogeneous blob objects through voting and level set formulation, Pattern Recognit Lett. 2007, 28(13).
[24] Sébastien Bougleux, Gabriel Peyré, Laurent D. Cohen. Anisotropic geodesics for perceptual. grouping and domain meshing. ECCV 2008.

[25] Matthew Cook, Probabilistic reasoning and decision making in sensory-motor systems. The Neuromorphic Engineer (2009).

[26] Ran Fan, Xiaogang Jin, and Charlie C.L. Wang, Multiregion segmentation based on compact shape prior. IEEE Trans. on Automation Science and Engineering, 2014.

[27] Tie Liu, Jian Sun, Nan-Ning Zheng, Xiaoou Tang, Heung-Yeung Shum. Learning to detect a salient object. In Proc. IEEE Cont. on Computer Vision and pattern Recognition (CVPR), 2007.

[28] N. Riche, M. Mancas, M. Duvinage, M. Mibulumukini, B. Gosselin, T. Dutoit, RARE2012: A multi-scale raritybased saliency detection with its comparative statistical analysis. Sig. Proc.: Image Comm. 28(6): 642-658 (2013).

[29] Hae Jong Seo, and Peyman Milanfar, Static and spacetime visual saliency detection by self-resemblance, J. Vision 9(12):15,1-27.

[30] R. Margolin, L. Zelnik-Manor, A. Tal. Saliency for image manipulation, The Visual Computer, 2012. 